\title{
Iqueye, a single photon-counting photometer applied to the ESO new technology telescope
}

\author{
G. Naletto ${ }^{1,2}$, C. Barbieri ${ }^{3}$, T. Occhipinti ${ }^{3}$, I. Capraro ${ }^{3}$, A. Di Paola ${ }^{4}$, C. Facchinetti ${ }^{5}$, E. Verroi ${ }^{1,3}$, P. Zoccarato ${ }^{3,6}$, \\ G. Anzolin ${ }^{7}$, M. Belluso ${ }^{8}$, S. Billotta ${ }^{8}$, P. Bolli ${ }^{9}$, G. Bonanno ${ }^{8}$, V. Da Deppo ${ }^{2}$, S. Fornasier $^{10}$, C. Germanà ${ }^{3}$, E. Giro ${ }^{11}$, \\ S. Marchi ${ }^{3}$, F. Messina ${ }^{9}$, C. Pernechele ${ }^{9}$, F. Tamburini $^{3}$, M. Zaccariotto $^{12}$, and L. Zampieri ${ }^{11}$
}

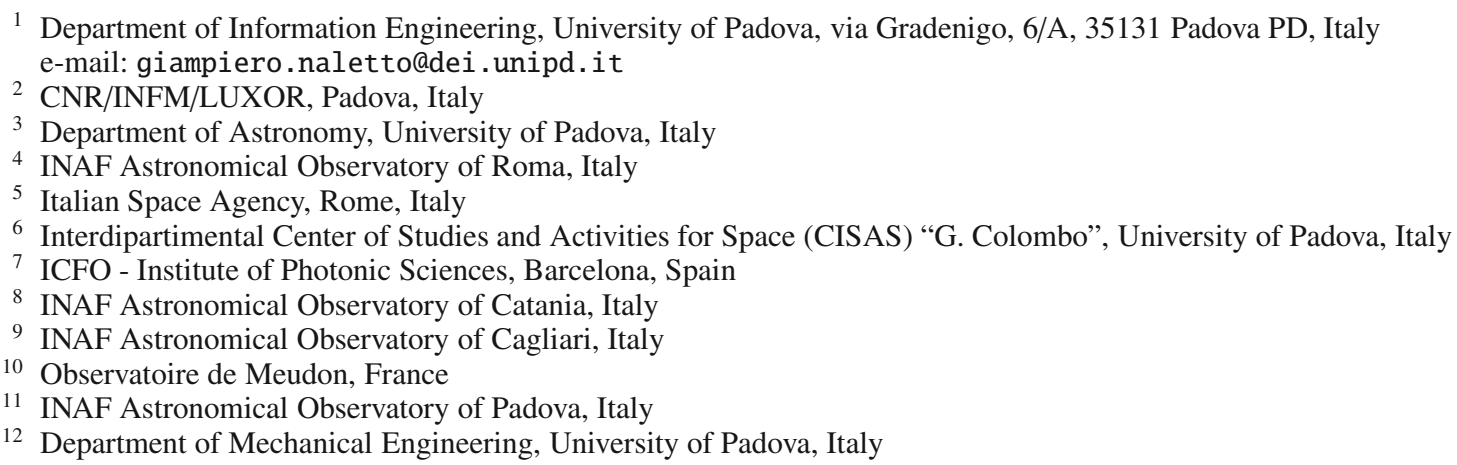

Received 10 July 2009 / Accepted 15 September 2009

\begin{abstract}
Context. A new extremely high speed photon-counting photometer, Iqueye, has been installed and tested at the New Technology Telescope, in La Silla.

Aims. This instrument is the second prototype of a "quantum" photometer being developed for future Extremely Large Telescopes of 30-50 m aperture.

Methods. Iqueye divides the telescope aperture into four portions, each feeding a single photon avalanche diode. The counts from the four channels are collected by a time-to-digital converter board, where each photon is appropriately time-tagged. Owing to a rubidium oscillator and a GPS receiver, an absolute rms timing accuracy better than $0.5 \mathrm{~ns}$ during one-hour observations is achieved. The system can sustain a count rate of up to $8 \mathrm{MHz}$ uninterruptedly for an entire night of observation.

Results. During five nights of observations, the system performed smoothly, and the observations of optical pulsar calibration targets provided excellent results.
\end{abstract}

Key words. instrumentation: photometers - techniques: miscellaneous - instrumentation: miscellaneaous

\section{Introduction}

Iqueye, the instrument described in the present paper, represents a second step in a program initiated in 2005. At that time, we had proposed the development of Quanteye (Dravins et al. 2005; Barbieri et al. 2006; Naletto et al. 2006) as a possible focal plane instrument of OWL, the $100 \mathrm{~m}$ OverWhelmingly Large Telescope (OWL 2005) of the European Southern Observatory. Quanteye was designed to be the fastest ever photon-counting photometer: it consisted of 100 parallel channels, each sampling a 1/100th portion of the telescope pupil and capable to time-tagging each detected photon with an accuracy of the order of $10 \mathrm{ps}$; the entire system was capable of handling up to $1 \mathrm{GHz}$ count rate continuously for hours. Quanteye represented a leap beyond current astronomical instrumentation, entering the new world of "quantum" astronomy. Current astronomical instrumentation essentially exploits only the properties of the first-order coherence of light, usually for imaging (spatial coherence) or spectroscopy (temporal coherence) investigations. However, by analyzing the second-order coherence functions
(Glauber 1963), additional information unattainable by classical means can be obtained; for example, details about the photon emission mechanisms of the source, i.e., whether the light originates in a thermal or laser source, or whether the photons were scattered between their source and the observer. A current limitation to carrying out this analysis is the not so large size of today's telescopes: in fact, very large photon fluxes are required to clearly differentiate the presence of a coherent component in the second-order correlation from photon-counting statistics. However, the amplitude of these second-order effects increases very rapidly with telescope aperture size (Dravins 2008): if Quanteye could be developed for the proposed Extremely Large Telescopes (ELTs), having a far higher flux of photons, because of its extremely high timing accuracy and the very long-term stability, it could be the first astronomical instrument to study these second-order correlation functions. Because of the optical solution for dividing the telescope aperture into 100 sub-apertures, Quanteye was understood to be capable of performing Hanbury Brown - Twiss intensity interferometry (Hanbury Brown 1974) over a large number of different baselines and in the blue 
region of the observed-frame optical spectrum. Even if OWL, now renamed the European ELT (E-ELT), has been downscaled to an aperture of $42 \mathrm{~m}$, the available photon flux still implies that Quanteye is an instrument of enormous potentialities. More details about the possible scientific return of a quantum photometer on an ELT, and of its unique capabilities on smaller size existing telescopes can be found in Dravins et al. (2006) and Barbieri et al. (2006, 2007a).

While awaiting the construction of E-ELT and to acquire experience with such novel type of astronomical instrumentation, we completed the first step of placing in operation a small prototype named Aqueye, the Asiago Quantum Eye (Naletto et al. 2007; Barbieri et al. 2007b, 2009b,c), at the Asiago (Italy) $182 \mathrm{~cm}$ telescope. Clearly, mounting this instrument on such a small telescope does not allow us to reach the astronomical quantum domain. However, the basic technology has been developed and applied, and the potentiality of the instrument has been demonstrated. In particular, we have demonstrated its ability to produce data of an exceptionally high dynamic range between a few tens of counts per second, limited by the system noise, up to $8 \mathrm{MHz}$, which is the maximum value at which the selected detectors operate in a linear regime. Furthermore, we have shown that the detection time of each photon can be assigned within 1 nanosecond, and that this performance can be maintained uninterruptedly for hours.

On the basis of these extremely positive results, we decided to proceed a step forward, by developing a second higher class prototype for a larger telescope. This new instrument named Iqueye, the Italian Quantum Eye, has been tested in January 2009 at the side B Nasmyth focus of the New Technology Telescope (NTT) on La Silla (Chile).

The following sections describe the main characteristics of Iqueye and provide examples of the results obtained. Section 2 describes the optical design, the adopted detectors, and the data acquisition system. Section 3 shows some example of the data acquired at NTT, with emphasis on the observation of optical pulsars. Finally, Sect. 4 provides a brief description of the already foreseen upgrades for Iqueye, and of possible future plans for an instrument of yet superior performance instrument to be mounted on $8 \mathrm{~m}$ class telescopes such as the VLT.

\section{Description of Iqueye}

Iqueye, as its precursor Aqueye, is a conceptually simple fixed aperture photometer that collects light within a field of view (FoV) of only a few arcseconds (selectable from $1^{\prime \prime}$ to $6^{\prime \prime}$ ), divides the telescope light beam into four equal parts, and focuses each sub-beam on an independent single photon-counting diode. Two wheels allow us to insert suitable filters and polarizers along the optical path. Since the instrument has no imaging capability, a field camera visualizes the portion of the sky under investigation.

The most innovative part of Iqueye consists of a data acquisition system that, coupled to ultrafast detectors plus a rubidium oscillator and a GPS receiver, allows us to time tag the detected photons with a final absolute UTC referenced rms time accuracy superior to $0.5 \mathrm{~ns}$ over one hour of observation.

We now describe the various components of Iqueye; first, the photon-counting detectors, then the optical subsystem, and finally the data acquisition and time-tagging subsystem.

\subsection{Photon-counting detectors}

The choice of the most suitable detector for this application was the main driver of Iqueye's design. We considered several photon-counting detectors, but none of them really fulfilled our scientific requirements. A photon-counting imaging-array detector placed at the telescope focal plane would have been the simplest and easiest to operate, but unfortunately there are many limitations in using this type of detectors. For example, image intensifiers coupled with either CCD or CMOS sensors do not allow a fast time-tagging of the detected events. MCP-based detectors (Datte et al. 1999; Siegmund et al. 2004, 2005), which potentially have extremely good temporal resolution down to a few tens of picoseconds, are limited by a fairly low maximum count rate of a few $\mathrm{kHz}$ and by relatively low efficiency photocathodes in the visible range. Even if the development of the second generation H33D MCP-based photon-counting detector (Michalet et al. 2006a,b) seems very promising (with an expected time resolution of the order of $250 \mathrm{ps}$, and global count rate of the order of $20 \mathrm{MHz}$ ), the presently available generation does not yet have the characteristics as advanced as required for Iqueye. Also, the novel arrays of single photon avalanche photodiodes (SPAD) (Niclass et al. 2008; Boiko et al. 2009), even if really performing in terms of efficiency, count rate, and timing accuracy, still have a very small fill-factor and low quantum efficiency.

Even state-of-the-art non-imaging photon-counting detectors are unable to achieve the performance required: photomultipliers have the drawback of needing high voltage and specific timing electronics, and are of relatively low quantum efficiency. SPADs have extremely good timing accuracy, can sustain fairly high count rates, have a resonably high quantum efficiency, and low dark count, but also have a small sensitive area that is usually very difficult to couple with a star image given by a large telescope.

After a detailed analysis of pro's and con's, we finally decided to adopt SPAD, which is definitely the most effective detector for our applications, in terms of time-tagging accuracy, quantum efficiency, and dynamic range. This choice also had some adverse consequences, in particular of finding an optical solution applicable to small sensitive areas (see Sect. 2.2). Even if Iqueye would then act as a fixed aperture photometer without imaging capability, so setting limitations on the possible scientific utilization, this was the only solution we could devise with the presently available technology and resources.

The selected Geiger-mode SPAD detectors are produced by the Italian company Micro Photon Devices (see Cova et al. 2004 , for a description of their characteristics). The benefits of these devices are numerous: in contrast to other photoncounting detectors, they can tolerate full daylight also when powered, a capability that makes much simpler all the integration and alignment activities; their quantum efficiency approaches $60 \%$ at $550 \mathrm{~nm}$, as measured in the laboratory of the Catania Observatory (Billotta et al. 2009); they are thermoelectrically regulated at a nominal temperature of $-10{ }^{\circ} \mathrm{C}$ to maintain a dark current lower than a few tens of counts per second; they have an almost flat BK7 window (the low optical power of this window does not alter the optical quality of the focused beam) that divides the active area from the ambient, allowing safe operations and avoiding condensation onto the sensitive area; this window is coated with high efficiency anti-reflection coating to enhance the system efficiency; the integrated timing circuit provides an extremely accurate signal, allowing a time accuracy of the order of 35 ps (or shorter); they are packaged in a robust box that allows easy handling, and their price is reasonably low. 


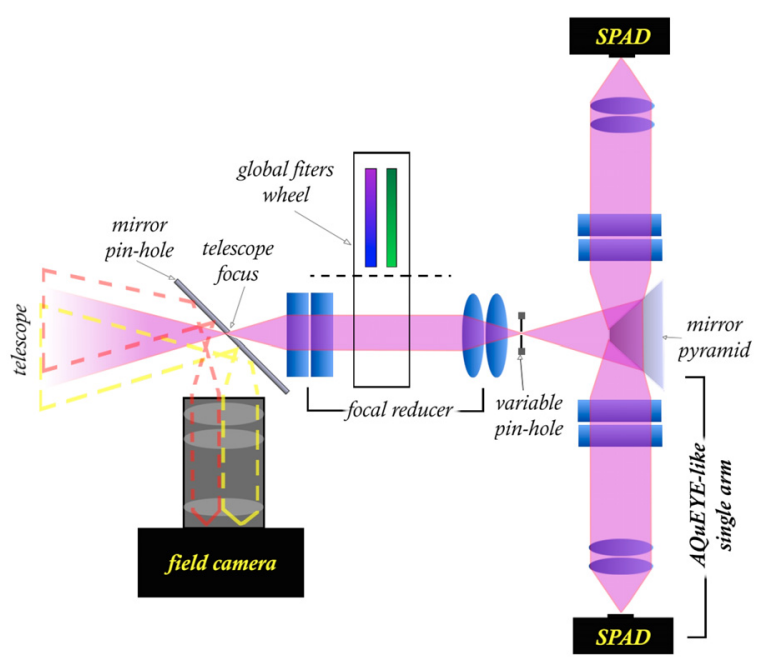

Fig. 1. Schematic view of Iqueye optical design.

The disadvantages are a small $100 \mu \mathrm{m}$ diameter sensitive area, a dead time of $\approx 75 \mathrm{~ns}$ after each detected event, a typical afterpulsing probability of around $1 \%$, and a linear count rate up to only $2 \mathrm{MHz}$ when using the timing accurate NIM output (the count rate linearity is as high as $12 \mathrm{MHz}$ with the TTL output, which however has a less accurate time-definition capability).

\subsection{Optical subsystem}

The optical design of Iqueye is fairly simple (see Fig. 1). At the NTT Nasmyth focus, where Iqueye interfaces the telescope, a holed folding mirror deflects the light from the telescope by $90^{\circ}$, sending, by means of a suitable objective, the field around the star to be studied in the field camera focal plane. The light from the target object instead passes through the hole and is collected by a collimating refracting system. Two filter wheels located after the first lens allow the selection of different filters or polarizers; the filters/polarizers presently available on these two wheels are listed in Table 1 . The light then reaches a focusing lens system, which, together with the preceding lenses, (de)magnifies the collected telescope image by a $1 / 3.25$ factor.

Within this intermediate focal plane, one of three pinholes (200, 300 and $500 \mu \mathrm{m}$ diameter, respectively) can be inserted. These pinholes act as field stops, and their size allows the selection of three different FOVs. Taking in account the $5.36 \mathrm{arcsec} / \mathrm{mm}$ nominal NTT scale factor at the Nasmyth focus, the diameters of the selectable FoVs are 3.5, 5.2, and 8.7 arcsec, respectively. After the pinhole, light impinges on a pyramid with four reflecting surfaces and whose tip coincides with the center of the shadow of the secondary mirror. This optical element divides the telescope pupil into four equal portions, and sends the light from each sub-pupil along four perpendicular arms. Along each arm, the sub-pupil light is first collimated and then refocused by a suitable lens system, which further (de)magnifies the image by an additional 1/3.5 factor, over the SPAD. Therefore, the SPAD circular sensitive area of $100 \mu \mathrm{m}$ diameter, nominally defines a FoV of 6.1 arcsec.

The smallest pinhole acts as a 3.5 arcsec field stop. This pinhole can be selected when it is necessary to reduce as much as possible the background around the target, as when observing a pulsar embedded in a nebula. The diameter of this pinhole is still compatible to seeing of the order of 1 arcsec rms; poorer seeing

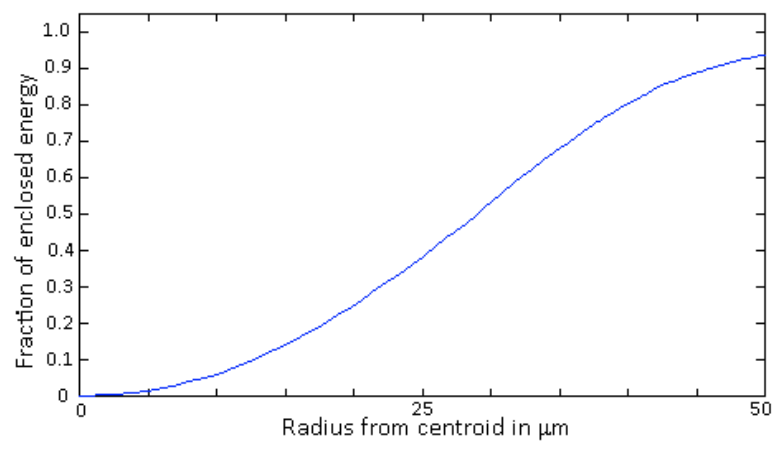

Fig. 2. Encircled energy plot showing the energy distribution on the $100 \mu \mathrm{m}$ diameter detector in the case of a polychromatic uniformly illuminated FoV of 5 arcsec.

values or telescope oscillations greater than \pm 1 arcsec manifest themselves as fluctuations of the detected signal. The intermediate 5.2 arcsec pinhole is more closely matched to the both detector and seeing, and also accounts for the effects of the small spot broadening caused by system residual aberrations. This is therefore the pinhole selected for the standard operating condition. Finally, the larger 8.7 arcsec pinhole is used mainly for alignment and/or calibration purposes.

It is clear that the optical requirements of Iqueye are not really stringent, since it behaves like a focal reducer. In addition, splitting the beam into four parts causes a reduction of a factor four in the optical invariant: the reduction in the aperture size lowers the speed of each arm, and consequently the system aberrations, thus simplifying the optical design. Because of this simplification, it has been possible to create the optical train using only commercial spherical lenses, yet still obtaining almost optimal performance. As an example of the simulated optical performance, Fig. 2 shows the plot of the encircled energy on the $100 \mu \mathrm{m}$ diameter SPAD with a polychromatic (four wavelengths being considered at 420, 520, 620 and $720 \mathrm{~nm}$ ) and uniformly illuminated FoV of 5 arcsec. This case is definitely worse than expected in normal conditions, where the light has an approximate Gaussian distribution with a $F W H M$ given by a typical seeing value of about 1 arcsec, and yet the energy encircled in the SPAD sensitive area is more than $94 \%$. It is clear that improvements are possible. The percentage of light focused on the detector is not much greater than $97 \%$ even in the case of a point source, because of the residual aberrations of commercial lenses; an optimized lens design would allow us to collect a percentage of radiant energy greater than $99 \%$.

Another important advantage of splitting the beam into four parts regards the detector characteristics. As mentioned in Sect. 2.2, the SPADs used have a $75 \mathrm{~ns}$ dead time, i.e. after the detection of one photon event, the detector is insensitive for this time interval. The dead time limits the dynamics to higher rates when observing a bright source, and is particularly severe for quantum applications, where photon statistics must be measured. The introduced parallelism between the sub-pupils, and the consequent use of four detectors, partially overcomes this problem. Because of the statistical distribution of events over the four detectors, many events that would be otherwise lost are recovered. This effect can be clearly seen in Fig. 3, where the two cases, single detector and four-detectors, are compared. The curves are histograms of the time differences between successive photon counts, binned into units of $1 \mathrm{~ns}$. The bottom curve shows the partial recovery of the otherwise lost counts at time intervals shorter than the dead time itself. Obviously, the larger 
Table 1. Main characteristics of the filters/polarizers available on the two Iqueye filter wheels.

\begin{tabular}{ccccc}
\hline \hline Position & Wheel 1 & Peak $(\mathrm{nm}) / F W H M(\mathrm{~nm})$ & Wheel 2 & Peak $(\mathrm{nm}) / F W H M(\mathrm{~nm})$ \\
\hline 1 & B & $433 / 101$ & Neutral 1 & - \\
2 & V & $531 / 82$ & Neutral 3 & - \\
3 & $\mathrm{R}$ & $747 / 234$ & HeII & $468 / 2$ \\
4 & IR & $817 / 179$ & OI & $630 / 2$ \\
5 & O[III] & $501 / 1.31$ & Pol Vis & Peak extinction ratio $>1: 1000$ \\
6 & H $\alpha$ & $656 / 3$ & Pol UV & Peak extinction ratio $>1: 100000$ \\
7 & Shutter & - & Neutral 2 & - \\
8 & Clear & - & clear & - \\
\hline
\end{tabular}
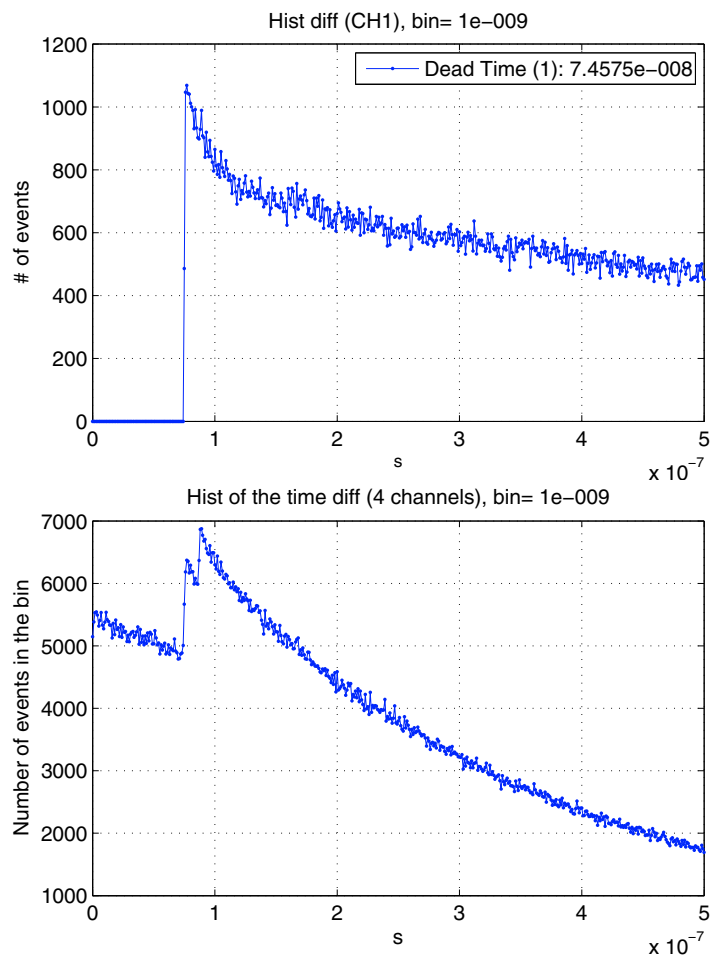

Fig. 3. (Top) Measurement of the dead time of one SPAD. (Bottom) Dead time for the combination of the four SPADs, showing a partial recovery of the lost counts at time intervals shorter than the dead time itself.

the number of detectors, the smaller is the overall effect of the dead time.

The global efficiency of the system has also been estimated by taking into account the three reflections of NTT mirrors, the reflection at the pyramid, the nominal transmission of all the lenses, and the detector quantum efficiency. The result obtained is shown in Fig. 4, together with the expected sensitivity when using the three $B, V$, and $R$ wide-band filters. It can be seen that the global sensitivity curve is dominated by the SPAD efficiency (Billotta et al. 2009) reaching a peak of more than $33 \%$ at $550 \mathrm{~nm}$.

From this curve, it is also possible to evaluate the expected signal-to-noise ratio (SNR) as a function of the target magnitude. As an example, Fig. 5 shows the calculated exposure times needed to achieve the required SNR for a given exposure time, in the $V$-band, per single SPAD, assuming a source at zenith, no moon light, a sky brightness of $21.9 \mathrm{mag} / \operatorname{arcsec}^{1}$, a collected FoV of 3 arcsec, and a detector dark count rate of $50 \mathrm{c} / \mathrm{s}$. At the

${ }^{1}$ Data for La Silla were taken from: http://www.vt-2004.org/ gen-fac/pubs/astclim/lasilla/index.html

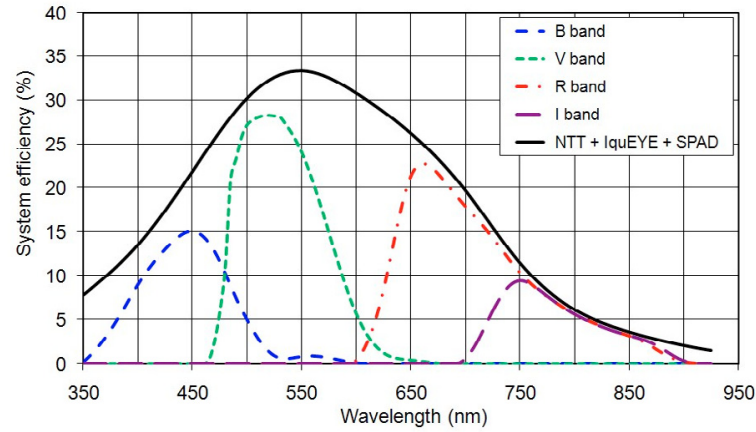

Fig. 4. Estimated global efficiency of Iqueye applied to NTT.

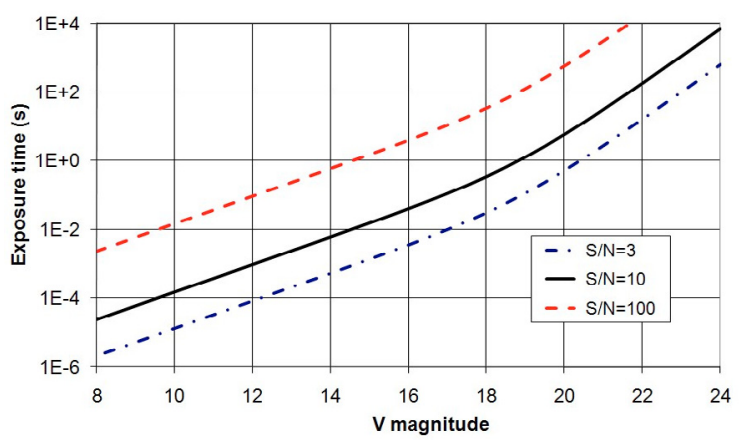

Fig. 5. The calculated exposure time to reach a wanted SNR in the $V$ band.

faintest magnitudes, Iqueye SNR is limited by the detector dark count and not by the night sky.

\subsection{Iqueye data acquisition system}

The task of the Iqueye data acquisition system (IDAS) is conceptually very simple: it must collect pulses produced from each SPAD when a photon is detected, assign a very accurate absolute time tag to each event, and store the time tags in the external memory. In reality, this is not an easy task at all, because of the stringent requirements: namely, to produce an absolute, UTC referenced time tag for each detected photons with a rms accuracy of the order of $500 \mathrm{ps}$ for at least one hour of continuous operation, coping with count rates ranging from few tens of $\mathrm{Hz}$ up to $8 \mathrm{MHz}$.

The starting point for defining the IDAS (see Fig. 6) is the pulse produced by the SPADs once a photon is detected. As already mentioned, the SPAD used has two different outputs: a TTL one, which can sustain count rates as high as $12 \mathrm{MHz}$ in the linear regime, with about 250 ps timing accuracy and a NIM 


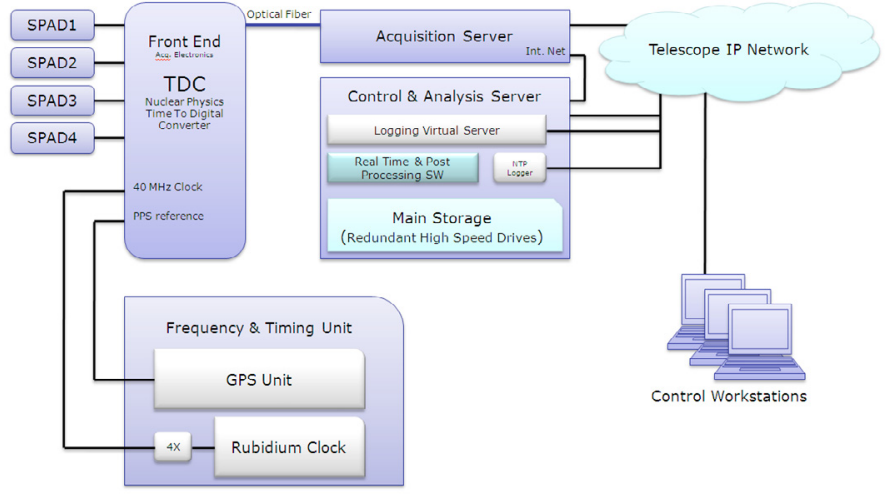

Fig. 6. Conceptual schematic of the acquisition and timing system of Iqueye.

one, which has a maximum linear count rate of about $2 \mathrm{MHz}$, but a much more accurate 35 ps typical timing accuracy. Since Iqueye was designed to reach a rms time tagging resolution of the order of $100 \mathrm{ps}$, the NIM output was adopted as a baseline, notwithstanding the lower possible maximum count rate, while the TTL one can be used in parallel mainly for control purposes.

Each of the 4 NIM signals is transferred from the SPADs, by means of calibrated equal electrical length coaxial cables to ensure the same electrical delay, to one of the input channels of a time to digital converter (TDC) board (CAEN V1290N, originally developed for high energy physics applications). This TDC board is nominally able to time-tag the voltage pulses on its inputs with a 24.4 ps time resolution: this very high sampling is obtained by means of a $40 \mathrm{MHz}$ internal oscillator whose frequency is multiplied by 1024 by means of a phase locked loop (PLL), and a delay locked loop (DLL), providing a $40 \mathrm{GHz}$ clock. Unfortunately, the quality of the internal oscillator is insufficient to satisfy the extremely severe stability requirement of our applications, for which it is necessary to obtain simultaneously both the short-term stability typical of a quartz oscillator and the long-term one assured by a primary time reference. This task could be reached by using primary time references such as hydrogen-maser clocks, which however are really expensive, difficult to handle and not available at NTT. To obtain a nearly optimal and affordable performance, we therefore focused our attention on a combination of a rubidium oscillator, a GPS receiver, and a post-processing algorithm, as described in the following.

The Iqueye time and frequency unit (ITFU), shown in Fig. 7, is based on the concept of correcting the long-term drift of the reference frequency provided by the rubidium oscillator by means of a post-processing algorithm that uses the pulse-persecond (PPS) signal provided by a mini-T Trimble GPS receiver. The GPS also provides the synchronization to UTC. The ITFU uses a Stanford Research System FS275 rubidium oscillator that produces a $10 \mathrm{MHz}$ sinusoidal signal, that then is converted to a $40 \mathrm{MHz}$ TTL signal by means of a frequency multiplication by a Tektronics AFG3251 pulse generator and a signal generator. The TTL signal represents the input to the IDAS. The rubidium atomic clock is the optimal trade-off in terms of performance and costs, ensuring good stability for medium lengths of time $\left(<10^{4} \mathrm{~s}\right)$. The most stable and high performance clock would be the hydrogen maser, but its cost exceeds the available resources.

As shown in Fig. 7, the rubidium oscillates in free-running mode, that is it is not disciplined by an external reference because this would worsen the phase noise. The tests performed

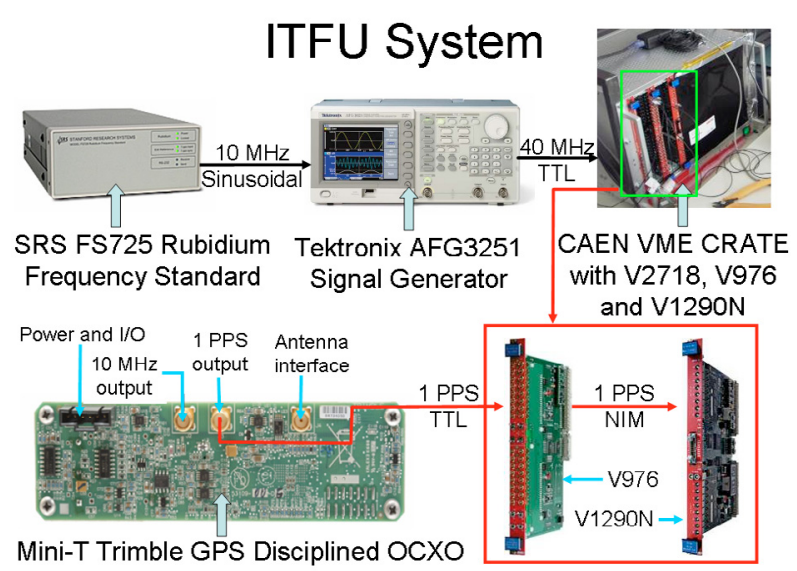

Fig. 7. Block diagram of the Iqueye time and frequency unit, which provides the frequency standard to the experiment. OCXO is an acronym for Oven-Controlled Crystal Oscillator.

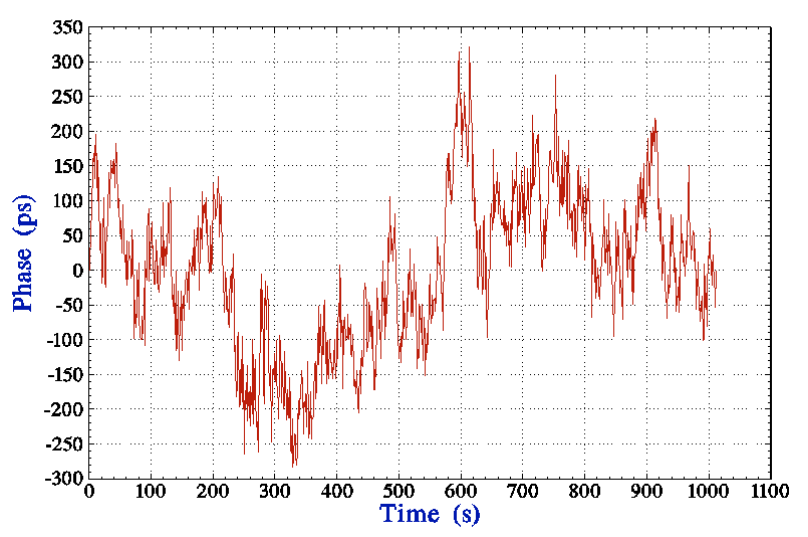

Fig. 8. Residuals of the rubidium oscillator pulse per second (PPS) versus the Astronomical Observatory of Cagliari caesium oscillator PPS after removal of the linear phase drift. The abscissa spans over $1000 \mathrm{~s}$.

at the Laboratory of Time $\mathcal{F}$ Frequency of the Astronomical Observatory of Cagliari (OAC) using a primary standard caesium clock as reference, showed that the rubidium accumulates a phase drift of approximately 65 ns after one hour, and $160 \mathrm{~ns}$ after two hours. However, by removing such linear drift, only a residual stochastic phase error remains, whose rms value is shorter than $50 \mathrm{ps}$ for a duration of more than $1000 \mathrm{~s}$ (see for example Fig. 8). For the Allan variance, it has a minimum corresponding to $2.5 \times 10^{-13}$ after $10^{4} \mathrm{~s}$.

By adding the rms time errors in the SPADs ( $35 \mathrm{ps}$ ), in the electronics chain (50 ps), and in the residual phase error (50 ps rms for one hour of observation) to the internal sampling discretization (24.4 ps), a total rms time error of approximately $100 \mathrm{ps}$ is obtained, which satisfies the design requirements. This is the level of relative rms time accuracy that we can reach with Iqueye for one-hour observations.

To remove the linear drift and obtain the UTC synchronization while operating at the telescope, Iqueye uses the PPS signal provided by a GPS receiver. These PPSs are given to the CAEN board via a TTL/NIM converter as input pulses on a fifth channel, and are processed by the board, in the same way as photon pulses from the 4 SPADs are treated. The CAEN internal oscillator is disabled, and the board uses the $40 \mathrm{MHz}$ frequency 
provided by the ITFU. Post-processing algorithms estimate and remove the rubidium oscillator phase drift using the GPS PPS, and finally the UTC corrected time tags are made available for further processing. To determine the uncertainty in the start time, the start signal is provided by the PC controlling the data acquisition, whose system clock is disciplined by the Network Time Protocol with a typical uncertainty of few milliseconds. However, the true start of the acquisition is given by the first PPS encountered in the data string, and therefore has a rms uncertainty of approximately $\pm 25 \mathrm{~ns}$ as typically associated with a good quality single frequency GPS receiver. However, owing to the linear drift interpolation, the error associated with the starting time decreases with the number of acquired PPS: by means of simple calculations, it can be verified that after one hour of observation this error decreases to $0.4 \mathrm{~ns}$ rms. Therefore, considering the 100 ps relative time accuracy for an acquisition of one hour, an absolute rms time accuracy with respect to UTC of shorter than $0.5 \mathrm{~ns}$ is obtained.

A limitation of the TDC board is that it returns a time-tag value digitized at 21 bits. Given the 24.4 ps time digitization, the longest unambiguous time interval is only $\Delta t=51.2 \mu \mathrm{s}$, while all times longer than this interval have an initial phase $N \Delta t$, where $N$ is an integer to be determined. To realize this socalled "de-rollover" process, we feed the TDC board with an external $20 \mathrm{kHz}$ reference pulse, which allows us to determine the value of $N$ and recover the initial phase.

The overall system was designed with a modular approach. The TDC board is equipped with 16 inputs: presently only six of them are used, so that a further expansion of the number of detectors is possible with no need to upgrade the IDAS. Moreover, the TDC board is lodged inside a standard crate, which can host additional boards all connected to the same VME bus. In practice, other TDC boards can be added, if necessary, with no need to modify the present system.

A dedicated server manages all the data exiting the TDC. This server is connected to the VME bus inside the CAEN crate. From here, through a bridge and an optical fiber, the data are sent to the acquisition server, with an approximate maximum speed of $60 \mathrm{Mb} / \mathrm{s}$. This is the present bottleneck that limits the maximum count rate of the system, but it can be overcome as discussed in the final considerations. The user interface, which is implemented by means of a second server, has been developed as a Java multitasking code: it allows the acquisition control and the data storage, the control of each instrument subsystem and in particular of all the mechanisms, it can perform several realtime quick-look statistical analyses of the acquired data, and allows us to read and store the images of the stellar field around the selected object acquired by the field camera. These images can be used for an a-posteriori analysis of guiding errors and sky conditions. The storage capability is approximately $2 \mathrm{~TB}$ : all the data are recorded in a 6 SATA disks raid6 configuration, to ensure both redundancy and speed in accessing the database. All the electronics equipment is mounted into a standard 19" rack that can be placed into the NTT Nasmyth focus room, just under Iqueye's optics. The whole system is connected to the NTT IP network, so that the instrument can be remotely controlled.

We remark that the stored data contain long strings of time tags. With these data, all possible types of post-processing analysis (i.e., calculations of autocorrelation, cross-correlation, FFT, power spectra, periodograms, light curves) can be done without affecting the integrity of the original data. For instance, the events can be sorted by the most convenient time bin for the specific scientific analysis without losing any original information.

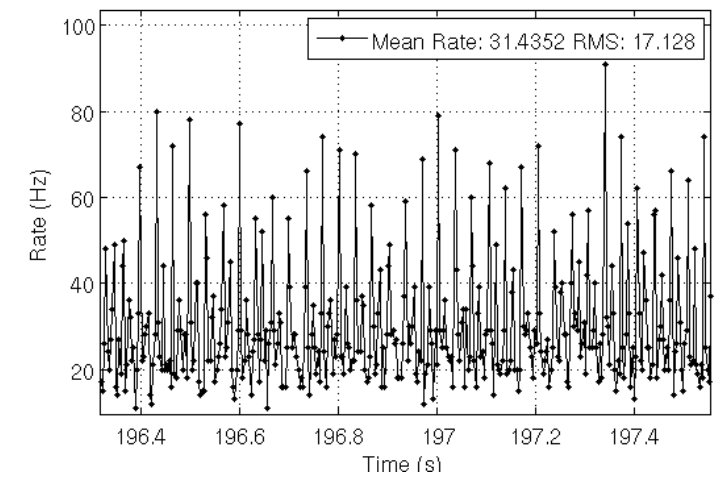

Fig. 9. Individual counts from the Crab Nebula pulsar, with $0.33 \mathrm{~ms}$ (1/10 of the period) bin size. The average count rate per time bin is given in the box.

The main characteristics and performance of Iqueye are summarized in Table 2.

\section{Preliminary astronomical results of Iqueye}

We performed our observations at the NTT on 14-19 January 2009, operating Iqueye across its entire range of count rates, from extremely faint to very bright sources. The capability of our apparatus to time-tag photons with extremely accurate precision over a very wide dynamic range was demonstrated convincingly. We provide two examples of the single photon capabilities, leaving discussion of the astronomical results to future papers. The first example, the pulsar of the Crab Nebula, illustrates the capabilities of Iqueye in conventional high speed photometry. Figure 9 shows a string of individual pulses from the pulsar after transmission through a linear polarizer, with the counts binned in $3.3 \mathrm{~ms}$ time bins, which is one tenth of the pulsar period. The great sensitivity of Iqueye is clearly evident: it is able to resolve to high accuracy the $33 \mathrm{~ms}$ single periods of this weak $V=16.5$ object. Figure 10 shows the Crab pulsar light curve obtained by folding only $1 \mathrm{~s}$ integration counts with a $33.627 \mathrm{~ms}$ period and two different time bins: $0.07 \mathrm{~ms}$ on the left, and 0.6 $\mathrm{ms}$ on the right. One second of time is more than sufficient to obtain reliable information of this light curve.

The second example illustrates how to quantum astronomy can be achieved by the calculation of $g^{(2)}$, i.e., the second order correlation function (Dravins et al. 2005; LeBohec et al. 2008; Foellmi 2009; Barbieri et al. 2009a) using the present technology of Iqueye. In particular, we verified the capability of Iqueye to perform the measurement of $g^{(2)}(d)$ as a function of the relative distance $d$ of the collecting apertures, thus confirming its effectiveness as an intensity interferometer (Hanbury Brown 1974).

The bright star $\zeta$ Ori (HD 37743, $V=1.8, \mathrm{SpT}=09.5 \mathrm{Ib}$, with emission lines) was observed on January 21st, 2009 at UTC (start) $01 \mathrm{~h} 06 \mathrm{~m} 24 \mathrm{~s}$ for $1 \mathrm{~h} 8 \mathrm{~m}$ through the wide band $B$ and neutral density 2 filters using the 5.2 arcsec pinhole. The star was chosen because it appears in the list of stars observed with the intensity interferometer at Narrabri (Hanbury Brown et al. 1974). The authors quoted a zero baseline correlation coefficient $C_{N}=0.60 \pm 0.06$, very far from the value of 1 expected from a single unresolved star. Their explanation of the difference was that it is caused by the limited the resolution of the stellar disk, which corresponds to an angular extent of the order of 0.5 mas for a uniformly illuminated disk. It is well known (see 
Table 2. Main characteristics describing the performance of Iqueye applied to NTT.

\begin{tabular}{ll}
\hline \hline System sensitivity & Photon counting \\
\hline Relative time accuracy & $100 \mathrm{ps}$ (for $1 \mathrm{~h}$ of continuous observation) \\
Absolute time accuracy & $500 \mathrm{ps}$ (for $1 \mathrm{~h}$ of continuous observation) \\
Dark count rate & $<100 \mathrm{~Hz}$ \\
Maximum count rate & $8 \mathrm{MHz}$ \\
Dynamic range & $>40 \mathrm{~dB}$ \\
Limiting magnitude & $m_{V}=24$ with 2 h exposure time and $S / N=10$ \\
Effective field of view & (selectable) $3.5,5.2$, or 6.1 arcsec \\
Operative spectral range & $\Delta \lambda=[350,925] \mathrm{nm}($ see Table 1 for filter details) \\
System total efficiency & $33 \%$ (peak @ 550 nm) \\
& $18 \%$ (average over $\Delta \lambda=[350,925] \mathrm{nm}$ spectral range) \\
\hline
\end{tabular}
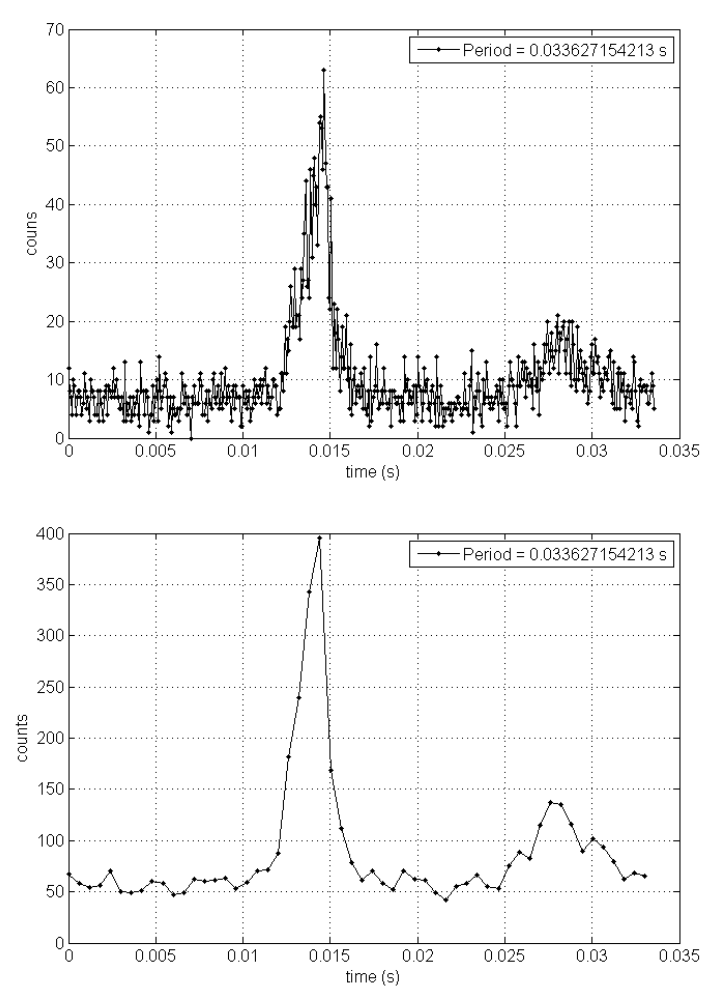

Fig. 10. Crab pulsar light curves obtained with one second of acquisition. The same data have been binned with two different time bins: $0.07 \mathrm{~ms}$ (top) and $0.6 \mathrm{~ms}$ (bottom).

Mason et al. (1998) and the Washington Double Star Catalog 2 ) that the $\zeta$ Ori is a triple star, the secondary $(\zeta$ Ori B, $V=4.20$, $\mathrm{SpT}=\mathrm{B} 0 \mathrm{III}$, flux $B / A \approx 0.1$ ) being approximately 2 '. 5 distant to the south east, and therefore just at the edge of the used pinhole. The tertiary weaker component is too far to be of concern in our data.

The method we have used to calculate $g^{(2)}(d)$ consists of correlating the event time-tag strings obtained by each SPAD, searching for coincidences among every pair of possible combinations. In effect, the photons collected by each SPAD arrive from four different sub-pupils of the NTT aperture: this allows us to consider the system as four parallel smaller telescopes, each focused on one SPAD. Clearly, the collecting area of each sub-aperture is small and the baseline distances $d$ are fixed even though they rotate on the sky during the exposure time, because of the telescope Alt-Az mount; we therefore did not expect to

2 The Washington Double Star Catalogue is maintained at the U.S. Naval Observatory http://ad.usno.navy . mil/wds/wds.html obtain significant astrophysical results. Since the separation between the telescope sub-apertures is small compared with the Narrabri telescopes, we expected that $g^{(2)}(d)_{\mathrm{NTT}}>g^{(2)}(0)_{\mathrm{NARR}}=$ $C_{N}$, and that $g^{(2)}(d)$ is slightly smaller than 1 . This was, first, because the stellar disk cannot be resolved by the small baselines available with this setup, and second, because the secondary star at the edge of the field of view should contribute a minimum amount of additional flux, since seeing is occasionally scattering a fraction of its light outside the pinhole. Finally, because the maximum overall photon rate that can be handled with the present equipment is approximately 10 times lower than the minimum rate necessary to achieve an acceptable signal-to-noise ratio (see Hanbury Brown 1974) for detecting second order effects. We therefore performed this experiment in preparation for an observation with two larger and more widely separated telescopes such as two VLTs, and and improved Iqueye now under construction. Nevertheless, we believe that these preliminary results are of sufficient interest to be expounded here because, to our knowledge, they represent the first attempt to perform intensity interferometry by time-tagging each detected photon and then post-processing the data, and thus avoiding real-time analog or digital correlators, which do not preserve data for all the original photon-events.

The method that we have followed to compute $g^{(2)}(d)$ values originates from (Beck 2007), who used two different coincidence detection techniques: one using time-to-amplitude converters, which is affected by dead-time problems as are our SPADs, and another using a logic circuit that has essentially no dead-time. The formula for the calculation of $g^{(2)}(d)$ is

$g^{(2)}(d)=\frac{N_{a b}}{N_{a} N_{b}} \frac{\Delta T}{\Delta t}$

where $N_{a}$ and $N_{b}$ are the total counts on detectors $a$ and $b$ respectively, and $N_{a b}$ is the number of coincidences, which is the number of simultaneous event detections in $a$ and $b$ within a coincidence window $\Delta t$ during an observation time $\Delta T$.

Determining the $N_{a}$ and $N_{b}$ values is straightforward, but this is not the case for $N_{a b}$. The calculation of the correlation between the channels is not a trivial task when the coincidence window $\Delta t$ is very small and the observing time $\Delta T$ is very long. To illustrate the difficulty, we consider sampling one hour of observations using the smallest coincidence window allowed by Iqueye, $24.41 \mathrm{ps}$, which corresponds using about $1.5 \times 10^{14}$ windows: after assigning one bit to any of these windows (" 0 " when there is no coincidence, " 1 " when there is a coincidence), each of the files to be correlated would have a size of about 18 TB.

We therefore devised an ad hoc Matlab algorithm based on the principle that if two events are separated by a time difference $m$, then the correlation vector at position $m$ must be increased by 1 . The algorithm was optimized to work with time 
Table 3. Values of $\left\langle g^{(2)}(d)\right\rangle$ obtained from one hour observation of the bright star $\zeta$ Ori, for each pair $a, b$ of sub-apertures, assuming a time coincidence window $\Delta t=244.1 \mathrm{ps}$.

\begin{tabular}{ccccc}
\hline \hline $\begin{array}{c}\text { Channel pair } \\
\text { (channel numbers) }\end{array}$ & $\begin{array}{c}\text { Effective distance } \\
d(\mathrm{~m})\end{array}$ & $\begin{array}{c}\text { Resolving angle } \\
\theta(\operatorname{arcsec})\end{array}$ & $\left\langle g^{(2)}(d)\right\rangle$ & $\begin{array}{c}\text { Standard } \\
\text { deviation }\end{array}$ \\
\hline A-B & 1.7 & 0.052 & 0.886 & 0.002 \\
A-C & 2.4 & 0.037 & 0.912 & 0.003 \\
A-D & 1.7 & 0.052 & 0.931 & 0.001 \\
B-C & 1.7 & 0.052 & 0.909 & 0.002 \\
B-D & 2.4 & 0.037 & 0.909 & 0.004 \\
C-D & 1.7 & 0.052 & 0.932 & 0.004 \\
\hline
\end{tabular}

bins of $24.41 \times 10^{N} \mathrm{ps}$, where $N$ is a non-negative integer, because these choices of bin-size minimize the computation time. As input, this routine needs the files that collect the time tags: even if the size of these data files remains large, the algorithm performs the calculation in a reasonable time, even without the use of supercomputers. In the specific case of $\zeta$ Ori, approximately $2 \times 10^{10}$ photons were detected, and the time tags were collected in 7771 files each of $10 \mathrm{MB}$, for a total of $78 \mathrm{~GB}$. Processing all these data with the smallest possible 24.41 ps coincidence windows lasted only about half a day on a $32 \mathrm{~GB}$ RAM four processor machine.

To obtain a clearer understanding of the results and in particular estimate the statistical errors affecting the determination of $g^{(2)}$, the entire string of 7771 files was divided into seven clusters of 1000 files each (the remaining 771 files were discarded), and the value of $g^{(2)}(d)$ was calculated for each cluster by setting a coincidence window $\Delta t=244.1 \mathrm{ps}$. From these values, the average $\left\langle g^{(2)}(d)\right\rangle$ was calculated together with its standard deviation. The results obtained from this analysis are summarized in Table 3. The table shows the pairs of SPADs used in the calculation, each SPAD being represented with a capital letter; not all combinations are shown because in the cross-correlation, pairs A-B and B-A give identical results. The flux-weighted distance $d$ between the two apertures is given in the second column, and the third column provides an indicative resolving angle computed as $\theta \approx \lambda / d$, where the effective wavelength $\lambda=430 \mathrm{~nm}$ has been taken to be the center of the observing filter. The last two columns indicate the value of $\left\langle g^{(2)}(d)\right\rangle$ and its standard deviation respectively. Note that the last column is not the true error in the value of $g^{(2)}(d)$, but simply the standard deviation of the average value $\left\langle g^{(2)}(d)\right\rangle$, calculated by considering the seven independent 1000 file clusters.

It is clear that the measurement and the algorithm are very stable, with relative fluctuations of $g^{(2)}(d)$ smaller than $1 \%$. In addition, when all the 7771 files are processed together, the $g^{(2)}(d)$ values obtained differ from those of the 1000 clusters by less than $1 \%$. Although the results are internally consistent, and even given values slightly less than 1 as expected a priori, we recall that this analysis was not designed to provide a true measurement, but simply to validate the method. The small aperture of the telescope and the already mentioned limitation in the dynamic range of the electronics used in January 2009, provided a signal-to-noise ratio too low to achieve significant astrophysical results.

\section{Future developments}

During three years of laboratory and telescope work, we have shown the advantages of using single photon avalanche diodes operated in Geiger mode for high time-resolution astrophysical problems. In particular, the superior quality of the La Silla site and the performance of NTT have allowed us to obtain a significant amount of scientific data that is now being analyzed. The detectors we have employed have shown good performances in terms of quantum efficiency, time tagging accuracy, high dynamic range, modest dark count, reliability and ease of operation. In contrast, its disadvantages include a the single-pixel small-area configuration, a long dead-time preventing us from exceeding a count rate of approximately $14 \mathrm{MHz}$ per SPAD, and a non-negligible afterpulsing effect.

Several improvements remain possible for Iqueye. Iqueye is now being fitted with custom lenses to achieve a higher concentration of the light on the SPAD. In addition, four filter wheels, one per SPAD, are being added, to allow simultaneous multispectral analysis. The throughput at the NIM connector could be increased toward the theoretical limit imposed by the dead time, which could also be shortened; MPD is already attempting to find the optimal compromise between deadtime, timing jitter, and afterpulsing specifically for the foreseen astronomical applications. In conjunction with CAEN, we propose to develop a dedicated TDC board with the acquisition computer directly inside the VME crate. The technology of single photon detectors is also rapidly improving, driven by other powerful users such as the community of classical and quantum communications.

We note that the instrument we have described, suitably adapted, could be immediately applied to the VLT, in particular for HBT intensity interferometry. However, by the time that the E-ELT is ready, photon-counting arrays with imaging capabilities may be available, greatly improving the performance of this type of instrumentation. As a final consideration, accurate time-keeping is a fundamental variable for this type of applications: first class observatories, in particular the VLT and the E-ELT, should be equipped with primary time standards as all VLBI stations are.

\section{Conclusions}

The main conclusions of our present study can be summarized as follows.

1. A new extremely fast photon-counting photometer, Iqueye, has been tested at NTT, where its superb potentiality for observing fast variable objects, such as pulsars, was clearly demonstrated. These tests permitted hours of uninterrupted observations with an absolute timing accuracy superior to $0.5 \mathrm{~ns}$.

2. Iqueye paves the way toward the realization of a truly "quantum" photometer for the $42 \mathrm{~m}$ E-ELT.

3. The results already obtained by Iqueye attest that a quantum photometer at the E-ELT will be able to measure second and higher order correlation functions, so opening the road to new tools of investigation of the Universe. 
Acknowledgements. Iqueye was constructed with funds provided by the Italian Ministry of University and Research, the University of Padova, and the National Institute for Astrophysics through the PRIN 2006 program; by the European Community through the Harrison Project for the scientific exploitation of the Galileo GNSS; and by Fondazione CARIPARO through the Project of Excellence 2006. The authors wish to express gratitude to ESO who granted technical time at NTT for testing Iqueye and covered part of the team travel expenses. Finally, a sincere acknowledgement is due to La Silla personnel, who participated very actively in the observational campaign and made a substantial contribution to the success of the instrument testing.

\section{References}

OWL Concept Design Report 2005, Tech. rep., ESO document OWL-TRE-ESO0000-0001 Issue 2

Barbieri, C., Da Deppo, V., D’Onofrio, M., et al. 2006, in The scientific requirements for extremely large telescopes, ed. P. Whitelock, B. Leibundgut, \& M. Dennefeld, IAU Symp., 232, 506

Barbieri, C., Dravins, D., Occhipinti, T., et al. 2007a, J. Mod. Opt., 54, 191

Barbieri, C., Naletto, G., Occhipinti, T., et al. 2007b, Mem. SAIt. Suppl., 11, 190

Barbieri, C., Daniel, M., de Wit, W., et al. 2009a, New Astrophysical Opportunities Exploiting Spatio-Temporal Optical Correlations, Science white paper prepared for the Astro2010 Decadal Review, available at [arXiv:0903.0062]

Barbieri, C., Naletto, G., Occhipinti, T., et al. 2009b, J. Mod. Opt., 56, 261

Barbieri, C., Naletto, G., Verroi, E., et al. 2009c, in Science with the VLT in the ELT Era, Astrophysics and Space Science Proceedings, 249

Beck, M. 2007, J.O.S.A., 24, 2972

Billotta, S., Belluso, M., Bonanno, G., et al. 2009, J. Mod. Opt., 56, 273

Boiko, D., Gunther, N., Brauer, N., et al. 2009, New J. Phys., 11, 013001 (7 pages)

Cova, S., Ghioni, M., Lotito, A., Rech, I., \& Zappa, F. 2004, J. Mod. Opt., 51, 1267
Datte, P., Birkbeck, A., Beuville, E., et al. 1999, Nucl. Instrum. Methods Phys. Res. A, 421, 576

Dravins, D. 2008, in High Time Resolution Astrophysics, ed. D. Phelan, O. Ryan, \& A. Shearer, Ap\&SS Library, 315, 95

Dravins, D., Barbieri, C., Da Deppo, V., et al. 2005, QuantEYE quantum optics instrumentation for astronomy. OWL Instrument Concept Study, Tech. rep., ESO, Document OWL-CSR-ESO-00000-0162

Dravins, D., Barbieri, C., Fosbury, R., et al. 2006, in The scientific requirements for extremely large telescopes, ed. P. Whitelock, B. Leibundgut, \& M. Dennefeld, IAU Symp., 232, 502

Foellmi, C. 2009, A\&A, submitted for publication, abstract available at http: //arxiv.org/abs/0901.4587

Glauber, R. 1963, Phys. Rev., 131, 2766

Hanbury Brown, R. 1974, The intensity interferometer. Its applications to astronomy (London: Taylor \& Francis)

Hanbury Brown, R., Davis, J., \& Allen, L. 1974, MNRAS, 167, 121

LeBohec, S., Barbieri, C., de Wit, W., et al. 2008, in Optical and Infrared Interferometry, ed. M. Schöller, W. C. Danchi, \& F. Delplancke, SPIE Proc., 7013, 70132E

Mason, B., Gies, D., Hartkopf, W., et al. 1998, AJ, 115, 821

Michalet, X., Siegmund, O., Vallerga, J., et al. 2006a, in Optical Biopsy VI, ed. R. Alfano, \& A. Katz, SPIE Proc., 6092, 141

Michalet, X., Siegmund, O., Vallerga, J., et al. 2006b, J. Mod. Opt., 54, 239

Naletto, G., Barbieri, C., Dravins, D., et al. 2006, in Ground-Based and Airborne Instrumentation For Astronomy, SPIE 6269, 62691W-1/9

Naletto, G., Barbieri, C., Occhipinti, T., et al. 2007, in Photon counting applications, Quantum Optics, and Quantum Cryptography, SPIE, 6583, 65830B-1/14

Niclass, C., Favi, C., Kluter, T., Gersbach, M., \& Charbon, E. 2008, IEEE J. of Solid-State Circuits, 43, 2977

Siegmund, O., Vallerga, J., McPhate, J., \& Tremsin, A. 2004, in UV and GammaRay Space Telescope Systems, ed. G. Hasinger, \& M. Turner, SPIE Proc., 5488, 789

Siegmund, O., Vallerga, J., Jelinsky, P., Weiss, S., \& Michalet, X. 2005, IEEE Nuclear Science Symposium Conference Record, N14-55, 448 